\title{
Troubles sexuels résultants de la stérilité (après échec des traitements)
}

\author{
Frédérique GUILLET-MAY, Olivier THIEBAUGEORGES, Nadia DANDACHI, \\ Annie ZACCABRI, Patricia BARBARINO-MONNIER, Odile HEYMES, Claire BUSSELOT
}

Unité fonctionnelle d'A.M.P.,Clinique Universitaire de Gynécologie Obstétrique, Nancy

RESUME

A côté des troubles sexuels à l'origine d'une stérilité, on recense lors des échecs de prise en charge de couples stériles en Aide Médicale à la Procréation (AMP) ou lors de l'inclusion dans des protocoles d'insémination avec sperme de donneur (IAD) l'apparition de dysfonctions sexuelles. Les réactions affectives à l'échec de traitement sont comparables à un processus de deuil, mais avec perte d'un projet de vie potentielle, et souvent deuil de la sexualité.

Face au diagnostic de stérilité définitive, on recense tant chez l'homme que chez la femme 70 à $80 \%$ de dépressions réactionnelles, troubles sexuels, et difficultés relationnelles.

L'équipe d'AMP de Nancy retrouve 13 dysfonctions sexuelles sur 33 couples en échec de Fécondation In Vitro (FIV) et 15 dysfonctions sexuelles sur 120 couples en IAD ; elle détaille les conduites à tenir préventives.

Dans tout parcours d'AMP, la qualité de la vie sexuelle doit être interrogée par les médecins ; les antécédents de violences sexuelles seront recherchés ; les limites temporelles de la prise en charge doivent être régulièrement fixées.

La prise en charge multidisciplinaire (gynécologue obstétricien, andrologue, psychiatre, psychologue, sophrologue, infirmier et sage-femme) est la seule garante d'une prévention de troubles psycho-sexuels résultant des traitements de stérilité.

Mots clés : stérilité, sexualité, psychosomatique, désir sexuel, qualité de vie, dysfonction érectile

\section{INTRODUCTION}

Les prises en charge de stérilité et les parcours en Aide Médicale à la Procréation (AMP) sont souvent très longs. Les examens sont parfois invasifs, tant chez la femme (hystéroscopie, cœlioscopie, laparotomie...) que chez l'homme (cure de varicocèle, biopsie testiculaire...). Ensuite, peuvent se succéder des cycles d'inséminations avec sperme de conjoint ou de donneur (IAD) et/ou des cycles de fécondation in vitro (FIV) avec ou sans microinjection ou encore avec sperme de donneur. II n'est pas rare qu'au terme de ces thérapeutiques, l'enfant attendu ne soit toujours pas là.

Pour certains couples stériles, l'impossibilité de recours à l'aide médicale à la procréation en intra-conjugual est considérée d'emblée comme un échec du fait de la nécessité de recourir au sperme de donneur (voire au don d'ovocyte ou au don d'embryon que nous n'envisagerons pas ici).

Ces situations sont difficiles pour les couples mais également pour les médecins. Le médecin, en effet, sait proposer des thérapeutiques et les accompagner mais l'arrêt de la prise en charge est plus malaisé pour lui. II lui faut du courage, des arguments et un savoir dire après une écoute empathique. Le pire consiste à poursuivre les thérapeutiques, quand le couple attend, sans le dire, l'arrêt de la prise en charge. Ceci nécessite une bonne expérience $[3,1]$.

Correspondance :

Dr Frédérique GUILLET-MAY - UF d'AMP,

Clinique Universitaire de Gynécologie Obstétrique,

Rue du Docteur Heydenreich, 54042 NANCY Cedex -

Tél 03.83.34.44.26 - Fax 03.83.34.44.02 -

Email f.guillet-may@maternite.chu-nancy.fr 


\section{PRINCIPAUX TROUBLES PSYCHO-SEXUELS SECONDAIRES A LA PRISE EN CHARGE DE LA STERILITE}

Si les troubles de la sexualité peuvent être à l'origine d'une stérilité, ils peuvent aussi survenir lors de sa prise en charge [13].

Face à l'échec de la procréation intra-conjugale peuvent survenir des troubles de l'érection, l'absence d'orgasme chez la femme, une baisse du désir et de la fréquence des rapports sexuels vécus comme inutiles. Le couple a dû accepter le clivage sexualité-reproduction. II a donc dû se réorganiser [3].

Les hommes et les femmes ne sont pas positionnés au même endroit ; ce sont les femmes qui portent les enfants (!). Et le discours médical n'est pas reçu de la même façon selon les sexes [9].

Les réactions affectives à l'échec des traitements de la stérilité sont souvent comparables au processus de deuil avec cinq étapes :

- choc et dénégation ;

- colère ;

- marchandage et les patients deviennent exigeants, désirant l'impossible ;

- dépression ;

- acceptation.

La différence par rapport au deuil, c'est qu'il s'agit cette fois de la perte d'un projet de vie potentielle d'une part et que ce deuil de la fertilité s'accompagne souvent d'un deuil de la sexualité.

En outre, la phase d'acceptation a été repoussée durant les explorations et les traitements.

Les réactions au diagnostic de stérilité définitive, d'après Micioni [12] consistent en dépression réactionnelle, troubles sexuels et difficultés relationnelles (Tableau 1).

Les patients, spécialement en cas de stérilité inexpliquée, croient souvent que leur stérilité est d'origine psychogène quand les traitements échouent.

Ce diagnostic de stérilité définitive et d'absence possible de toute thérapeutique peut détériorer la relation du couple par manque de communication. Les femmes surtout s'en plaignent, mais dans la consultation, les hommes verbalisent

Tableau 1 : Aspects psychologiques de la demande d'IAD (d'après Micioni [12]).

\begin{tabular}{lll}
\hline & HOMMES & FEMMES \\
\hline Diagnostic bien toléré & $29,8 \%$ & $35,7 \%$ \\
Dépression réactionnelle & $42,5 \%$ & $36,4 \%$ \\
Troubles sexuels & $11,9 \%$ & $12,5 \%$ \\
Difficultés relationnelles & $9,3 \%$ & $8,2 \%$ \\
\hline
\end{tabular}

leur souffrance, ce qu'ils ne peuvent faire dans l'intimité conjugale [9].

L'isolement social se surajoute souvent, alors que respect, compassion et compréhension de l'entourage, s'ils ne résolvent pas les problèmes, consolent.

\section{L'EXPERIENCE DE L'UNITE D'AMP DE NANCY}

En 1994, dans l'équipe d'AMP de Nancy, 33 couples en échec de FIV et 120 couples en demande d'IAD ont été reçus par le psychiatre sexologue de l'Unité [14].

Treize dysfonctions sexuelles ont été recensées dans le premier groupe : dysfonctions érectiles primaires ou secondaires coïncidant avec la 1ère FIV, vaginisme, absence de relation sexuelle par absence de désir et crainte de l'échec.

Dans ce premier groupe on recense :

- 10 cas d'angoisse ;

- 9 cas de dépression ;

- 3 cas de conflits conjugaux.

Chez les 120 couples vus avant inséminations avec sperme de donneur, on recense :

- 15 dysfonctions sexuelles ;

- 2 dépressions ;

- 1 conflit conjugal patent.

Depuis 1998 , le psychiatre de l'équipe n'est plus sexologue mais pédopsychiatre, car il travaille principalement en maternité.

Durant les entretiens pré-IAD, la problématique sexuelle n'est plus jamais abordée, l'entretien est centré essentiellement sur l'enfant à venir et le secret.

\section{VIE SEXUELLE ET INSEMINATIONS AVEC SPERME DE DONNEUR (IAD)}

Si le psychiatre n'aborde pas le problème des relations sexuelles en IAD, il faut espérer que tous les gynécologuesobstétriciens évoquent avec le couple la qualité de leur vie et en particulier de leur vie sexuelle lorsqu'il pose l'indication d'IAD $[2,10]$.

Face au diagnostic de stérilité définitive d'origine masculine, les réactions des hommes, les plus fréquentes sont:

- un vécu persécutoire et agressif par rapport aux parents, aux médecins ;

- des idées ou des gestes suicidaires;

- des sentiments de culpabilité envers la femme avec parfois proposition de séparation.

Les réactions féminines les plus habituelles sont :

- une négation de la dépression pour soutenir le partenaire;

- un sentiment de culpabilité face au désir d'enfant et à sa propre fertilité : 
- une prise en charge de la responsabilité de la stérilité visà-vis de l'entourage ;

- une agressivité envers le partenaire, intériorisée, ayant pour conséquence des difficultés interpersonnelles.

Après que le gynécologue ait proposé l'IAD, la décision est souvent renvoyée d'un partenaire à l'autre en raison de la peur d'offenser le partenaire, de blesser l'orgueil masculin. Le plus souvent c'est le mari qui propose l'IAD comme acte de réparation envers sa femme et lui-même. Chaque partenaire essaie de se mettre à la place de l'autre plutôt que de recentrer sur lui-même en tant qu'homme ou en tant que femme.

La dysfonction sexuelle est le symptôme de tout ce qui se joue à ce moment là. L'évoquer avec le couple permet d'aider à comprendre pourquoi le désir s'étiole devant ces bouleversements psychiques.

\section{GUIDE DE BONNES PRATIQUES POUR LA PREVENTION DES TROUBLES SEXUELS CHEZ LES COUPLES STERILES APRES ECHEC DES THERAPEUTIQUES OU AVANT IAD}

Le gynécologue-obstétricien et l'andrologue doivent systématiquement ouvrir la discussion sur la qualité de la vie sexuelle, comme élément de qualité de vie au sens de I'OMS. Non seulement, il convient de poser la question de la fréquence des rapports sexuels mais aussi de leur qualité et d'introduire la notion de plaisir [8]. Si la procréation peut être considérée comme le superlatif de la santé, le corps stérile lui est vécu comme un tombeau (Eberhardt, philosophe).

En cas de troubles sexuels, les médecins doivent poser la question des antécédents de violences sexuelles. La consultation de stérilité est souvent l'occasion d'une première révélation. Les dysfonctions sexuelles font partie intégrante du syndrome post-traumatique après viol et ce drame doit pouvoir être verbalisé afin que procréation et enfantement se passent dans de bonnes conditions [6].

II faut interroger chaque partenaire sur la cause des troubles pour eux, et corriger, et informer. Le mémoire de MarieLaure Gamet en sexologie a révélé que $37 \%$ des femmes enceintes après FIV étaient en demande d'informations sur la sexualité au cours de leur grossesse [5].

II convient de proposer une aide individuelle ou au couple, par sexothérapie, psychiatrie, psychologie ou sophrologie $[7,9]$.

Il faut éviter de négliger l'homme stérile, surtout dans les processus d'IAD où non seulement son sperme est écarté, mais sa vie sexuelle ignorée $[11,15]$.

Pour prévenir les effets psycho-sexuels néfastes de l'arrêt du traitement $[4,6]$ :

- chaque membre de l'équipe doit garder à l'esprit la fin de la prise en charge, à n'importe quel stade et connaître les réactions du couple ;
- le gynécologue doit redire les limites de la médecine de la procréation, expliquant ainsi que l'échec n'est pas d'origine psychogène ;

- dire que l'équipe a fait tout ce qui était en son pouvoir, et le couple aussi ;

- oser évoquer la vie sans enfant ;

- savoir questionner les couples sur ce qui leur a paru négatif au cours du traitement, ce qui pourrait aider d'autres couples. Cette question apporte souvent des doléances des hommes sur le peu de place qui leur est fait dans nos équipes.

- prendre du temps de consultation pour terminer une prise en charge, proposer un suivi gynécologique autre et une aide psychothérapique. Aménager des espaces de paroles avec d'autres couples ou avec les infirmières ou les sages-femmes des équipes.

\section{CAS CLINIQUE}

Monsieur et Madame F. nous consultent après un premier échec de Fécondation in vitro. Cette FIV survenait après une stérilité primaire de 6 ans, prise en charge par une autre équipe. Le vécu de cette tentative était à l'évidence, vu l'attitude du couple, très mauvais. Le couple ne se regarde pas, ne se parle pas. L'interrogatoire révèle que Madame F. s'ennuie et trouve que sa sexualité n'est plus ludique depuis la FIV. Le patient révèle une dysérection depuis la prise en charge en Fécondation in vitro. Nous leur proposons une aide par la sexologue de l'équipe. Au cours des 5 à 6 consultations du couple ou individuellement de l'homme et de la femme, Monsieur réussi à travailler sur la "distance " et sur la redécouverte de sa partenaire comme une femme à désirer.

La patiente quant à elle travaille sur la différence masculinféminin, sa capacité à porter un enfant et sa culpabilité de ne pas "accrocher" les embryons implantés en FIV. Après ces consultations de sexologie, le couple souhaite l'arrêt de la prise en charge, non seulement sexologique, mais également de la Fécondation in vitro. Le patient se dit satisfait car il peut parler avec sa compagne. Et la patiente dit que sa vie sexuelle est plus épanouie et plus spontanée et décide de débuter une thérapie analytique.

\section{CONCLUSION}

Seule une prise en charge multidisciplinaire (Figure 1) en parcours d'aide médicale à la procréation permet un abord curatif, mais aussi préventif des troubles sexuels secondaires à la stérilité. La discussion collégiale des cas difficiles, permet de transmettre les éléments importants de cette réflexion à plusieurs, aux couples et ainsi de les aider ; mais elle aide également les médecins à aborder l'échec auquel ils n'ont pas été formés et auquel ils sont souvent confrontés en médecine de la reproduction. 


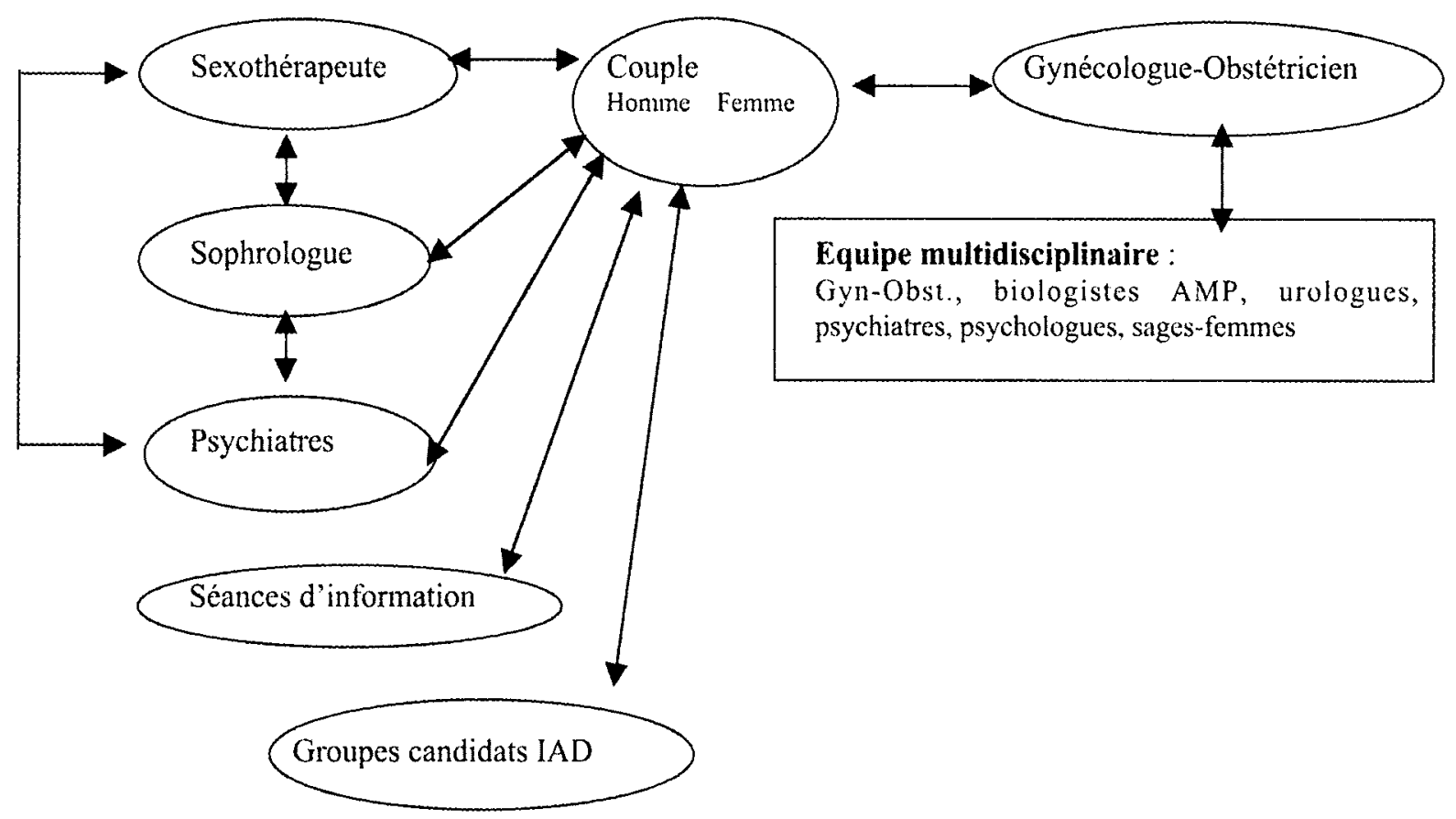

Figure 1 : Prise en charge multidisciplinaire des couples stériles avec dysfonction sexuelle.

\section{REFERENCES}

1. BONIERBALE M. : Couple et dysfonction sexuelle. $\left.X\right|^{e}$ Rencontres Nanceiennes de Gynécologie-Obstétrique. 12 octobre 2001, Nancy.

2. GUILLET-MAY F., CHEVRET-MEASSON M., DUDKIEWICZSIBONY M., LACHOWSY M. : "Psy" et qualité de vie du couple. Journée nationale de la Fertilité. 11 décembre 1999, Paris, Association Pauline et Adrien.

3. De BRANCION M.M. : La médecine de la procréation compromet la fécondité du désir. In : Sexe et Guérison. Paris, L'harmattan, 1998 : 227-239.

4. DARVISCH J. : Sexuality. In : Guidelines for Counselling in Infertility. Eshre, Monographs. Oxford University Press, 2002 : 27-28.

5. GAMET M.L. : De l'intérêt de parler de sa sexualité à une femme enceinte. Mémoire pour le Diplome Inter-Universitaire de Sexologie. Toulouse, Messages, $2002: 1-40$.

6. GUILLET-MAY F. : Le médecin face aux agressions sexuelles et au viol. Encycl. Med. Chir. (Elsevier Paris) AKOS. Encyclopédie Pratique de Médecine, 1999. 3-1410 : 1-5.

7. HEYMES O. : Sophrologie ou proposition d'accompagnement en secteur d'aide médicale à la procréation. Mémoire de formation à la gynécologie psychosomatique 1992 - 1995, APHP.

8. KENTECHI H. : Introduction : Guidelines for Counselling in Infertility. Eshre Monographs, Oxford University Press, 2002 : 13.

9. KLOCK S.C., MAIER D. : Guidelines for the provision of psychological evaluations for infertile partients at the University of Connecticut Health Center. Fertil. Steril., 1991, $56: 680-685$.

10.LEROY-BILLIARD M. : Qualité de vie et traitement de l'infertilité. Rev. Fr. Gynécol.Obst., 1999, 94, 2 : 107-110.

11.MAHLSTEDT P., GREEFELD D. : Assisted reproductive technology with donor gametes : the need for patient preparation. Fertil. Steril., 1989, 52 : 906-914.
12.MICIONI G., JEKER L., DE VITAS S. et al. : Aspects psychologiques de le demande d'IAD. J. Gynecol. Obstet. Biol. Reprod., 1985, 14 : 695-702.

13.MIMOUN S. : Les multiples interactions entre l'infertilité et la sexualité. Contracept. Fertil. Sex., 1993, 21 : 251-254.

14.REICHENBACH S., GUILLET-MAY F. : Dysfonctions sexuelles et procréation médicalement assistée. Rencontres Nancéennes de Gynécologie Obstétrique, Octobre 1994, Nancy.

15.SHENFIELD F., STEELE D.J. : What are the effects of anonymity and secrety on the welfare of the child in gamete donation? Hum. Reprod., 1997, 12 : 392-395.

Communication aux Journées de la FFER, Montpellier, septembre 2003. Manuscrit reçu : janvier 2004 ; accepté : février 2004.

\section{ABSTRACT}

\section{Sexual disorders resulting from sterility treatments}

\section{Frédérique GUILLET-MAY, Olivier THIEBAUGEORGES, Nadia DANDACHI, Annie ZACCABRI, Patricia BARBARINO-MONNIER, Odile HEYMES, Claire BUSSELOT}

Sexual dysfunction can cause sterility, but the failure of IVF (In Vitro Fertilization) or Sperm Donor Insemination (SDI) sterility treatments can also be responsible for sexual dysfunction. 